placed between the iron rings in order to produce the depolarizing field $H$.

With $H=0$, the polarization effect was found to be $(0 \cdot 65 \pm 0 \cdot 28)$ per cent of the total count (of which about one half was due to thermal neutrons). With $H=4$ gauss, the polarization effect was negative, but at the limit of error $(-0 \cdot 36 \pm 0 \cdot 31)$ per cent. At $H=2$ gauss an intermediate value of $(+0 \cdot 29 \pm 0 \cdot 36)$ per cent was obtained. These results are in qualitative agreement with the assumption $\mu_{n}=2$ N.M. The errors given are mean square errors; similar figures were obtained on computing the errors in the traditional way from the fluctuations of the single results. Altogether, $1 \cdot 2$ million counts have been made.

A new and improved apparatus is being prepared in order to determine the amount and the sign of $\mu_{n}$; the sign can be found by placing polarizer and analyser at an angle and by studying the direction of precession depending on the direction of the magnetic field.

Institute of

Theoretical Physics,

Copenhagen. April 7.

${ }^{1}$ Bloch, Phys. Rev., 50, 259 (1936).

Hoffman, Livingston, Bethe, Phys. Rev., 51, 214 (1937).

'Estermann, Stern, Z. Phys., 85, 17 (1933).

- Kellogg, Rabi, Zacharias, Phys. Rev., 50, 472 (1936).

' Estermann, Stern, Phys. Rev., 45, 761 (1934).

${ }^{6}$ Powers, Beyer, Dunning, Phys. Rev., 51, 371 (1937).

\section{Radioactive Neon}

IN a previous note ${ }^{1}$ it has been reported that the radioactive helium formed by bombarding finegrained beryllium hydroxide with neutrons can be transferred by a stream of gas through a tube from the place of activation around a neutron source to a Geiger counter placed at some distance.

By bombarding sodium or magnesium with fast neutrons, a radioactive substance of 40 sec. halfvalue period is produced ${ }^{2}$, which is generally assumed to be ${ }_{10}^{23} \mathrm{Ne}$. So far as I am aware, no chemical proof has been obtained. It therefore seemed of interest to attempt to use the above method to decide whether the active substance in question is a gas. Magnesium hydroxide was precipitated together with ferric hydroxide in various proportions by the method of $\mathrm{O}$. Hahn ${ }^{3}$ in order to give it a high emanating power. The dried precipitates were bombarded with neutrons from a beryllium-radon source of 200 millicuries, and air from an aspirator was passed at a suitable rate through the samples and then through a glass tube to the thin-walled jacket around the counter. No definite effect was found; it follows from the relative yields by bombardment of beryllium and magnesium with neutrons, that if radioactive neon is given off by the bombardment of magnesium, one would expect only a very weak effect (just detectable) by this method. This is mainly due to the small relative abundance of ${ }_{12}^{26} \mathrm{Mg}$.

In the case of sodium, the effect to be expected is much larger if the radioactive neon can be removed from the crystals of the sodium compound during an interval comparable to 40 sec. Commercial sodium stearate ${ }^{4}$ proved suitable, and an unambiguous effect was found. In Fig. 1, the number of counts per minute is plotted against the rate of the air stream in cubic centimetres per second. From the volumes of the activation chamber, the connecting tube and the jacket around the counter, one can calculate the shape of the curve to be expected when the radioactive gas has a given half-value period $T$. The curve drawn in the figure corresponds to $T=40$ sec. The experimental points are not in disagreement with this, although a slightly smaller value of $T(35-40$ sec.) would fit a little better. With sodium butyrate, a similar effect was obtained.

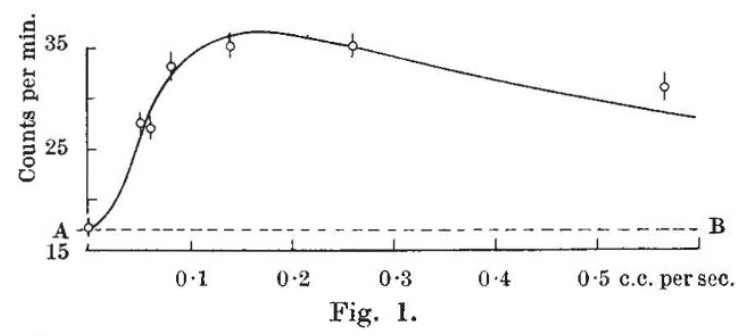

RELATION OF NUMBER OF COUNTS TO RATE AT WHICH THE AIR STREAM PASSED TO THE COUNTER. AB REPRESENTS THE $\gamma$-RAY EFFECT.

The effect here described may be of interest for the study of the emanating power of sodium com. pounds.

Institute of Theoretical Physics,
Copenhagen.

T. BJERGE. March 19.

${ }^{1}$ NATURe, 138, 400 (1936).

2 Fermi, Amaldi, D'Agostino, Rasetti and Segré, Proc. Roy. Sor., A 146, 483 (1934).

'Hahn, O., Naturwiss., 17, 295 (1929).

4 Strassmann, F., Z. phys. Chem., B, 28, 362 (1934).

\section{The Cosmological Constants}

Prof. P. A. M. Dirac's recent letter in Nature ${ }^{1}$ encourages me to direct attention to certain 'coincidences' which I had noticed some years ago, but which I have been hesitating to publish from the conviction that purely 'dimensional arguments' will not lead one very far.

If we consider the natural constants $h$ (Planck's constant), $c$ (velocity of light), $H$ (mass of the proton), $G$ (the constant of gravitation), we can form the following combination $M_{a}$ which is of the dimension of mass :

$$
M_{a}=\left(\frac{h c}{G}\right)^{\alpha} \frac{1}{H^{2 \alpha-1}},
$$

where $\alpha$ is an arbitrary numerical constant. Now a particular case of the above occurs in the theory of stellar interiors, namely, when $\alpha=3 / 2$. Then

$$
M_{3 / 2}=\left(\begin{array}{l}
h c \\
\dddot{G}
\end{array}\right)^{3 / 2} \frac{1}{\bar{H}^{2}} \doteq 5 \cdot 76 \times 10^{34} \mathrm{gm} .,
$$

which is about thirty times the mass of the sun. Now, the apparent success of steady state considerations in 'explaining' the observed order of stellar masses can be traced to the circumstance that the above combination (2) of the natural constants gives a mass of the correct order. It may be noticed that apart from numerical constants, (2) is the same as the upper limit to the mass of completely degenerate (degenerate in the sense of the Fermi-Dirac statistics) configurations ${ }^{2}$. The occurrence of (2) in stellar structure equations need not cause any surprise, 